dinarien viel deutlicher werden, den nur ein professoraler "Lernprozeß * und eine dadurch vermittelte $*$ Demokratisierung $\propto$ der Hochschulverfassung erweitern könnte. Doch davon ist man bei Professoren der Jurisprudenz noch weit entfernt.

Somit sind auch die Möglichkeiten einer von Studenten praktizierten Studienreform begrenzt. Ständige Disziplinierungsmaßnahmen fordern ihre Opfer. Die Herkunft der Studenten und eine noch immer am Positivismus orientierte Ausbildung, die zudem kein unmittelbar praktisches und wissenschaftliches Interesse, sondern ein Examensinteresse leitet, mahnen zur Vorsidht gegenüber Annahmen, die von einer schnellen Verhaltensänderung dieser Studenten ausgehen. Folgendes läßt sich jedoch konstatieren:

I. Arbeitsgruppen kritischer Studenten ist es möglich, einen Privatbereich zu verlassen und, sei es mit Hilfe der Institution Fachschaft, das Bewrußsein von Kommilitonen zu problematisieren.

2. Es liegt im Bereich des physisch und intellektuell Möglichen, daß diese Gruppen, bei der "Studienberatung* angefangen, eine Anpassung von Anfängern an den tradierten Studienbetrieb verhindern können und diese Situation auch zu stabilisieren vermögen.

3. Es ist ferner möglich - und das ist vor allem eine Frage der Taktik - die Fixierung einer Vielzahl von Studenten auf den Ordinarius zu brechen, um damit eine weitergehende Problematisierung von Lehrstoff etc. und eine selbsttätige Praxis vorzubereiten.

"Aber um sich zu heben, genügr es nicht, sich in Gedanken zu heben, und über dem wirklichen, sinnlichen Kopf das wirkliche, sinnliche Joch, das nicht mit Ideen wegzuspintisieren ist, schweben zu lassen « (Marx).

Heinricht Schacht

\title{
Seine Studenten und seine Sorgen
}

Am Mittwoch, dem 3I. Juli 1968, war Ernst Wolf „Gast der WELT*. Seine Äußerungen betitelte er $»$ Die Mißstände im Jura-Studium $\star$, wobei das erklärend hinzugefügte Motto "Meine Studenten und meine Sorgen* offenbar die Autorität des Mitleidenden bekräftigen soll.

Wolf ist Direktor des Instituts für Arbeitsrecht an der Philipps-Universität zu Marburg und einer der wenigen kämpferischen Initiatoren jenes nach eben diesem Ort benannten Manifests.

Dreierlei macht seinen Artikel bemerkenswert:

Die Diagnose, es gäbe an der Universität $»$ Mißständeк.

Die scharfe Einsicht in deren wahre Ursachen.

Die völlige Exkulpation der Professoren - zumindest als Ensemble einzelner Pädagogen.

Geprüft werden soll, was eine Analyse solcher Art leistet, ob sie eher erklärt oder verschleiert. Von dieser Aufgabe sollten nicht gelegentliche unflätige Randbemerkungen ablenken, die nur zum Ziel haben können, Ernst Wolf - ganz sicher vergeblich - lächerlich zu machen.

-Die Probleme, mit denen die Studenten ... zu kämpfen haben « kann man nicht 
auf ऋökonomische*, überhaupe nicht auf $*$ gesellschaftliche « und schon gar nicht

auf „politische« Gründe zurüdkführen. "Sogar sexuelle Gründe« - weitab von allem Gesellschaftlichen, versteht sich - werden bisweilen genannt. Das ist völlig abwegig.

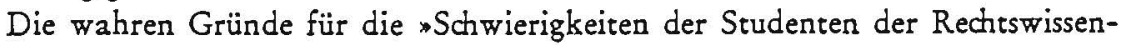
schaft, sich den juristischen Lehrstoff und die zur juristischen Arbeit notwendige Methodik anzueignen * sind *vorwiegend geistiger Art* und *haben seit längerer Zeit erheblich zugenommen «. Das führt zu den besagten »Mißständen «.

Ernst Wolf verteilt die Schuld daran recht gleichmäßig. Er dürtte vorher abgewogen haben. Wenn Studenten Ärger mit dem Lehrstoff haben, kann das sowohl am Lehrstoff als auch an den Studenten liegen.

Zum Lehrstoff

*In der deutschen Rechtswissenschaft hat sich seit dem Beginn dieses Jahrhunderts eine irrationalistische Grundeinstellung durchgesetzt, die nach dem ersten Weltkrieg für die Entwidklung bestimmend wurde, während des $>$ Dritten Reiches` kulminierte und heute noch vorherrscht. \& Die Phänomene dieser Entwicklung beschreibt Wolf korrekt: *Verzicht auf logische Methoden, begriffliche Ar-

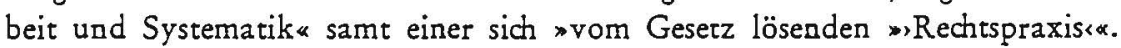
Die Rechtswissenschaft löst sich in »zahllose Spezialgebiere« auf. Auf den Lehrbetrieb bezogen ist eine »Aufblähung « des Lehrstoffes, seine zunehmende Strukturlosigkeir, gar eine fortschreitende Aufgabe der puristischen Terminologie zu konstatieren; $\gg . .$. ein inhaltsloses Vokabular wucherr ... «. Daraus resultieren die bekannten Kommunikationsschwierigkeiten zwischen Professoren und Studenten, was insbesondere daran liegt, daß die \... weitgehend aufgelöste Rechtswissenschaft ...* methodisch fremde Fragestellungen und Ausdrücke« unbesehen transplantiert - etwa »ökonomische «, »soziologische« und »theologische«. Das alles ist mißlich. Nur wäre es kleinlich, den Professoren »pädagogisches«Versagen vorzuwerfen, denn es hat sich als mächtiger Trend eben jene wirrationalistische Grundeinstellung * worwiegend geistiger Art* durchgesetzt. An diese wahre Ursache reichen auch Studienreformen nicht heran, sind sie doch nur als Notbehelf diskutabel und im übrigen »schon wegen der Zahl der Studenten « zu teuer. So darf die Existenz aus dem Wissenschaftsprozeß nicht zu legitimierender Herrschaftsverhältnisse unerwähnt bleiben.

Ernst Wolf weiß ein Heilmittel: Die "Neubegründung der Fachwissenschaften durch eine allgemeine Systematik der Wissenschaft und eine Rückkehr zu den Bedingungen objektiver Leistung ... . Dieser Appell an den guten Willen aller Einsichtigen ist von einer Blindheit gegenüber sozialen $Z$ wängen, die von einem 53 -jährigen Lehrstuhlinhaber wohl nur vorgespiegelt sein kann, sonst zeugre sie von unverfrorener Ignoranz.

Die wirrationalistische Grundeinstellung «, befällt sie den juristischen Geist wie Aussatz? Ihre auch von Wolf beobachtete Konstanz durch die politischen Systeme von Weimar bis Bonn hindurch sollte auf das verweisen, was an der Basis dieser Systeme ebenfalls prinzipiell konstant blieb - die Produkrionsverhältnisse im sich organisierenden Kapitalismus. So ist z. B. die Systematik des Schuldrechts im BGB noch auf einen ohne ökonomische Staatsintervention funktionierenden Markt gleicher Kontrahenten bezogen. Die heute das Schuldrecht überlagernden Allgemeinen Geschäftsbedingungen sind Ausdruck der wirtschaftlichen Beziehungen Ungleicher - und damit des Trends zur Monopolisierung. Diesem die formale Qualität des Rechts zerstörenden realen Trend dienten die Juristen - Richter oder Produzenten von Lehrmeinungen - zuerst in der Weimarer Republik als Vollstredker, indem sie sich ökonomischen Einflüssen eher beugten als Parlaments- 
gesetzen, in die das politische Gewicht ihrer Klassenfeinde - der Arbeiterparteien - mit eingegangen war.

Die objektiven $Z$ wänge bestehen fort. Der pure Wille zu einer neuen Systematik droht zu Bestrebungen zu gerinnen, die - wie weiland der Senatspräsident a. D. Baumbach - Gesetze vor der Verabschiedung einem Gesetzgebungsamt vorlegen möchten. Dort kann man sie unter dem Vorwand der formalen Aufmöbelung auf bornierte juristische Sozialideale hin umformen. Ernst Wolfs Blindheit gegenüber den Bestimmungsgründen der Entwidslung des Lehrstoffs hat ihre Entsprechung dessen Konsumenten gegenüber.

Die Studenten

Ihnen mangelt es immer mehr an "Kenntnissen * und "Denkschulung *. Das liegt an der Schule, die immer mehr Abiturienten mit zunehmend geringeren Leistungen ausstößr. Daran ist das die Hürden herabsetzende "Kultusministerium" schuld, denn $*$ Latein $\approx$ mit seinen hohen Anforderungen ans systematische Denken wird,.. zugunsten diffuser Fächer wie zum Beispiel, Gemeinschaftskundee reduziert..... Lateinlernen als eine der Grundvoraussetzungen einer neuen Systematik der Rechtswissenschaft? - das kann nur ein Witz sein. Zugunsten der umstrittenen Errungenschaft ,Gemeinschaftskunder ist zu sagen, daß - wenn dieses $>$ Fach، seinem Anspruch gemäß betrieben würde - es den Vorteil böte, die Wolfsche Blindheit heilbar zu machen. Doch Wolf insistierr: Ihm erzeugt die Schule zu viele undisziplinierte und leistungsschwache Abiturienten; es wird dort sogar »diskutierr «, natürlich sohne hinreichende Grundlagen «. Kein Wunder, daß man sich dann an der Universität schwer tut! *Bei solchen Verhältnissen kann es nicht überraschen, daß unter den Studenten eine Mißstimmung verbreitet ist, die von der geschickt arbeitenden Propaganda linksradikaler Gruppen für deren destruktive politische und igesellschaftspolitisches, das heißt marxistisch-gesellschaftsrevolutionäre Ziele erfolgreich ausgenutzt wird «.

Ernst Wolfs Analyse erklärt nichts. Ihre Geistesart ist symptomatisch für eine bestimmte Gattung von Professoren, deren Herrschaftsinteresse sie recht dilettantisch zu verschleiern sucht.

Anna Jobow

\section{Ein Flugblatt im besonderen Gewaltverhältnis}

Dem Assessor im Lehramt Heinz Lüdde, tätig am Kreisgymnasium in Heusenstamm (Landkreis Offenbach), wurde am Ir. November 1968 vom Regierungspräsidenten in Darmstade aus zwingenden dienstlichen Gründen die Führung seiner Dienstgeschäfte verboten ( $\$ 74$ Hessisches Beamtengesetz). Die sofortige Voliziehung der Suspendierung lag im öffentlichen Interesse ( $\$ 80 \mathrm{Abs.} 2 \mathrm{Ziff} .4$ Verwaltungsgerichtsordnung). Dieses wird repräsentiert durch zwei katholische Kirchenvorstände in Heusenstamm und einige Elternbriefe. Die zwingenden dienstlichen Gründe sind die Verteilung eines Flugblattes an Schüler einer 9. Klasse des Gymnasiums, die Erklärung des Assessors, die Lehrpläne für Schulen des Landes Hessen seien für ihn nicht verbindlich (bestritten) und die Veranlassung von Schülern, Resolutionen zu verfassen, in denen die Verhaltensweise bzw. der Unterricht älterer Kollegen kritisiert wurde (bestritten). $D_{a}$ die 